\title{
Du corps monstrueux au fantôme du corps : pudeur et impudeur comme modalités de construction d'un corps politique chez Hervé Guibert
}

\author{
Arnaud Genon \\ Nottingham Trent University
}

L'œuvre d'Hervé Guibert est l'histoire d'un corps. Elle est autre chose aussi, bien sûr, l'histoire d'un "je», une histoire de famille, d'amours, d'amitiés, l'histoire d'une époque, d'un regard... Mais indéniablement, le corps est ce qui offre au corpus sa cohérence puisque, selon les mots tout à fait justes de Ralph Sarkonak, le corps «n'est pas simplement un thème mais le principe générateur de l'œuvre» (1997, p. 9). Se penchant rétrospectivement sur l'ensemble de son travail, Guibert fait d'ailleurs le même constat, confiant à Christophe Donner : 
J'ai été frappé par l'introduction des Essais de Montaigne qui disait : « J'ai voulu me peindre nu », ça a fait tilt, je me suis dit que c'était quelque chose que je pourrais mettre en exergue à tout ce que j'ai fait, enfin de beaucoup de choses que j'ai écrites. J'ai eu l'impression par la force des choses d'être mon propre personnage, mais d'être aussi un corps mis en jeu dans des narrations, dans des situations, dans des rapports, j'ai aussi l'impression que c'est l'histoire d'un corps, effectivement d'un corps qui vieillit, d'un corps qui est malade, d'un corps qui est abîmé, d'un corps ceci, d'un corps cela, d'un corps qui renaît un peu, tu vois, d'un corps monstrueux aussi, d'un corps difforme, et j'ai l'impression que c'est l'histoire de ce corps. (1991a, p. 145)

Mais plus que du corps, il faudrait ici évoquer les corps... Ceux convoqués par Guibert sont tour à tour ceux d'amants, de ses grand-tantes octogénaires, ceux fantasmés dans des récits érotiques ou pornographiques - je pense précisément ici à la "plaquette pornographique » intitulée Les Chiens (1982) et au roman sadien Vous m'avez fait former des fantômes (1987) -, ceux en cire du musée Grévin, qui apparaissent dans plusieurs de ses photographies... Et lorsque l'on évoque le corps de l'auteur, qui nourrit et dévore en même temps la totalité de son œuvre, deux images m'interpellent particulièrement. Celle, tout d'abord, dans La Mort propagande (2009a), premier recueil de textes de l'écrivain, d'un corps monstrueux au sens où il se donne à voir, s'exhibe en toute impudeur, dans toute l'excentricité d'une recherche effrénée de plaisir. Un corps de jouissance, un corps à jouir. Exposé, appelé à être filmé, montré. À cette première figuration se substitue progressivement celle d'un corps amaigri, amoindri, souffrant, malade, qui n'est autre que le corps sidéen de l'auteur. Il s'expose dans un ensemble de postures qui le révèlent tout autant qu'elles le cachent, le dissimulent par l'intermédiaire de différents dispositifs autofictionnels : le je qui s'affirme est celui-là même qui se fond, 
se dissémine dans ses propres fictions de soi. Le corps guibertien, après s'être «outrageusement» affirmé, est alors amené à disparaître, à l'image de la dernière scène de son film, La Pudeur ou l'Impudeur (2009b), où il finit par quitter «la scène», fantomatique. La pudeur et l'impudeur ne constitueront en fait que deux modalités permettant la construction d'une image inédite du corps. C'est ce parcours dans l'œuvre de Guibert, à la recherche d'un corps qui joue sur différentes modalités de représentations, que je propose ici. Il s'agira alors d'une traversée chronologique qui visera aussi à mettre en lumière, dans une dernière partie, une politique du corps guibertien. Ce corps multiple, mis en spectacle, est aussi un corps fantôme en ce sens qu'il échappe à toute forme d'enfermement, de restriction : selon la formule de Michel Foucault, il n'est pas un «lieu sans recours auquel je suis condamné », mais se situe «toujours ailleurs, il est lié à tous les ailleurs du monde et à vrai dire il est ailleurs que dans le monde » (2009). Le corps qui s'écrit, qui s'inscrit dans les textes de l'auteur, se construit en dehors de tout repère, de toute norme sexuelle. Il refuse, nous le verrons, tout autant les modèles hétérosexuels qu'homosexuels, ancrant son corps dans un espace autre, incertain et indécidable. Un corps fuyant donc, recherchant dans les histoires qu'il raconte / qu'il se raconte tous les possibles d'une sensualité et d'une sexualité qui échapperaient à toute règle, à toute loi, à tout cloisonnement identitaire.

\section{Le corps de jouissance et l'esthétique de l'impudeur : une inscription autre du genre}

Avec La Mort propagande, initialement publié en 1977 aux éditions Régine Deforges, je me positionne aux origines du 
travail de Guibert, qui n'a alors que 21 ans et qui publie là son premier recueil de textes. Guibert commence ici son exploration du corps et son expérimentation littéraire, déjà appelées à se fondre l'une dans l'autre et à ne jamais se dissocier. Dans la lignée de Georges Bataille, de Pierre Guyotat, il offre un de ses textes les plus violents, crus et subversifs. Sans concession, sans pudeur, le narrateur y transforme son corps en laboratoire de jouissance et de souffrance qu'il offre en spectacle au lecteur, devenu malgré lui, comme dans le texte "L'œillade », voyeur et complice de ces mises en scène : " Mon corps est un laboratoire que j'offre en exhibition, l'unique acteur, l'unique instrument de mes délires organiques. Partitions sur tissus de chair, de folie, de douleur. Observer comment il fonctionne, recueillir ses prestations » (Guibert, 2009a, p.8). Cette Mort propagande constitue la mise en mots du projet que Guibert déclinera tout au long de son œuvre: décortiquer le corps, autopsier la pensée, les désirs, les fantasmes, n'en rien cacher, en prendre le risque et, comme le disait Leiris, «[m]ettre à nu certaines obsessions d'ordre sentimental ou sexuel, [... afin] d'introduire ne fût-ce que l'ombre d'une corne de taureau dans une œuvre littéraire » (Leiris, 2004, p. 10-11).

Le corps de Guibert, dans ce premier livre, est un corps liquide, une machine organique, une matière moite. Il est en même temps un réceptacle et un déversoir, un trou béant et un instrument à produire toutes sortes de substances expulsables... Qu'il y-a-t-il de plus impudique que de montrer le corps, de le révéler nu? Guibert nous répond ici : ce qu'il y a de plus intime que le corps, c'est ce qu'il recèle, ce qu'il cache, ce que l'on cache du corps en général et qu'il expose, lui, dans La Mort propagande comme une œuvre d'art, comme une offrande : « Un vrai corps, mon vrai sang. Prenez et mangez, buvez (ma 
paranoïa, ma mégalomania) », nous dit-il (2009a, p. 10). Ce qu'il y a de plus intime que le corps, c'est son intérieur, ce à quoi l'on a accès en passant par les cavités, où qu'elles se trouvent. Et pour exposer cet intérieur du corps impudique, Guibert commence par le situer dans « une salle de dissection » (2009a, p. 15), où il use de tout un arsenal médical : scalpel, lunette d'ophtalmologiste, petit ciseau, spatules, pinces recourbées ou incisives. Là, le corps peut être dépecé, autopsié, écartelé, couché, élargi, introspecté... Il s'agit bien d'exhiber, dès les premières pages, ce que le corps nu peut encore dissimuler : « les parois intestinales » (ibid.), « les plissés roses» (ibid. p.16) de l'anus puis ses «dédales compliqués, poches à crever, membranes, souterrains, glottes anales » (ibid. p. 17). Guibert donne de même accès à l'intérieur de son corps par l'intermédiaire de ce qui en sort. Ses déjections sont numérotées, photographiées, observées et, selon ses propres termes, « adorées comme des reliques divines » (ibid., p. 26). Le corps est aussi une mécanique olfactive, excitant l'imagination du narrateur. La crasse, les desquamations, les résidus urinaires qu'il mélange et se donne à sentir deviennent l'équivalent d'effluves orgiaques qui évoquent tous les vices.

Outre ces scènes solitaires d'auto-autopsies, de fétichismes en tous genres, le narrateur de La Mort propagande donne par ailleurs à lire ses relations sexuelles, décrites, elles aussi, sans fards. Il leur juxtapose des fantasmagories orgiaques dans lesquelles il inclut le lecteur par l'intermédiaire des pronoms de première et deuxième personnes du pluriel et par l'utilisation de verbes conjugués à l'impératif présent: « Ma bouche vous est offerte pour faire jouir vos bites [...]. Laissons le plaisir s'écouler, et spermons jusqu'à ce que nos couilles se rompent » (ibid, p. 63-64). On note dans ces passages qu'au plaisir de jouir 
se superpose une jouissance du dire, donnant l'impression que « la verbalisation devient la scène de la sexualité » (Butler, 2012, p.191). On touche à cette écriture présente dans ce que Barthes nommait le «texte de jouissance »: «celui qui met en état de perte, celui qui déconforte [...], fait vaciller les assises historiques, culturelles, psychologiques du lecteur, [...] met en crise son rapport au langage » $(1973$, p. $25-26)$. Il est vrai que Guibert, dans ces passages, fait preuve d'inventivité lexicale et surcharge les signifiants de signifiés: utilisation de mots plutôt rares tels les verbes "spermer», "gamahucher», de vocables appartenant au champ lexical de la médecine comme "gourmes» (maladie de peau), « chancres » (ulcération génitale) ou encore de néologismes du type «gourmons-les», qui signifie, dans le contexte, « transmettons-leur nos gourmes »...

Ce premier texte est fortement inspiré du roman $L e$ Nécrophile, de Gabrielle Wittkop (2001), mais aussi des peintures de Francis Bacon: «Il y avait également Francis Bacon. Parce que la peinture m'a autant marqué que la littérature. Les tableaux de Francis Bacon, c'était tout ce que j'aimais: la couleur, la violence, la boucherie, le corps, la sodomie, l'étreinte de deux hommes " (Guibert, 1991b). Ce recueil constitue en même temps l'ébauche de l'expression du corps dans le travail guibertien et son accomplissement le plus cru. C'est donc le caractère expérimental de La Mort propagande qui en fait sa force mais qui en révèle aussi les limites. Si l'enjeu est bien ici d'entreprendre un dévoilement de soi total, de "réduire cette distance entre les vérités de l'expérience et de l'écriture » (Guibert, 1988a), on comprend par ailleurs que la violence du dit vienne parfois opacifier le projet originel. Pour se dévoiler par l'écriture, il faut pouvoir être lu et, pour être lu, il faut être lisible. Je reprends ici le sens 
que Barthes donnait à ce terme lorsqu'il opposait le lisible au scriptible: le lisible englobe grosso modo les romans réalistes du $\mathrm{XIX}^{\mathrm{e}}$ siècle et les romans de masse du XXe siècle et s'oppose au scriptible, qui désigne une forme plus expérimentale de l'écriture, plus avant-gardiste, qui rompt avec les traditions, les habitudes de lecture et les processus d'identification. Or, force est de constater que Guibert construit un texte qui positionne le lecteur dans une difficulté, voire une incapacité à accepter cette représentation du corps. Je n'entends pas par-là que le corps représenté est inacceptable, qu'il est condamnable: il est juste difficilement recevable, car il est monstrueux en donnant à voir ce que tout le monde cache, en devenant un objet absolu de monstration.

On a souvent dit, et j'ai parfois moi-même avancé - mais pas à propos des textes que je viens de citer - que La Mort propagande constituait une tentative pour Guibert de trouver, de délimiter, voire de revendiquer une identité clairement homosexuelle. Toutefois, dans cette évocation de la jouissance, de sa jouissance, dans cette écriture clinique du corps, de son corps, même fantasmé et considéré comme lieu de plaisir, je ne vois plus désormais, à aucun moment, une quête ou une affirmation d'identité quelconque, mais plutôt la volonté de Guibert de se révéler à lui-même sa propre insaisissabilité. Le corps jouissant est un corps qui s'échappe, se lâche, se détend, se déprend. Ce corps offert, ce corps ouvert, disséqué, autopsié, est un corps que le narrateur lui-même n'arrive pas à saisir, d'où sa volonté, souvent répétée, de tenter de l'appréhender de manière détournée, médiatisée. Guibert use ou pense user d'une caméra : «Filmer mon cul en fonctionnement » (2009a, p. 9), note-t-il, ou souhaite se « donner la mort sur scène, devant les caméras» (ibid, p.10). Cette tentative / tentation de saisissement du corps jouissant est ailleurs médiatisée par un 
appareil photographique: "Je photographierai ma bite sous tous les angles, molle et tiède, dure et chaude, de face, de profil, recouverte de fourreaux divers : main, laine, cuir, huile, bouche, savon, pied d'éléphant en éponge fendu vulvé, tire-jus, pisse, merde et puis ton cul, ce cône à folie » (ibid, p. 22). Plus bas, il mentionne : «je me mis à photographier mes déjections » (ibid, p. 25). Cette représentation du corps jouissant est rendue possible plus tard par le prisme d'un miroir («Une glace est juste en face du siège [...]. Il se regarde jouir » (ibid, p. 54), ou encore par l'intermédiaire de l'autre, le voyeur qui saisit ce qui échappe au jouisseur lui-même : «Un voyeur patient a imaginé une stratégie. Non seulement la porte est discrètement trouée à hauteur de la vulve lorsqu'elle s'ouvre, mais la fenêtre [...] a été lacérée en plusieurs endroits » (ibid, p. 53).

Cette esthétique du gros plan, de l'impudeur donc, est de nouveau mise en œuvre, cinq ans plus tard, en 1982, dans Les Chiens, que Guibert qualifia lui-même, dans Fou de Vincent, de "plaquette pornographique ». Ce récit, écrit pour plaire à Michel Foucault, comme l'écrivain le confia à Didier Eribon (Guibert, 1991b), évoque d'abord les corps fantasmés d'un couple hétérosexuel faisant l'amour derrière une cloison où se trouve le narrateur. Il imagine les positions sexuelles qu'ils adoptent, leur corps à corps érotique : "leur commerce dans ma tête n'a pas de cesse et harasse mon corps, je reste debout, immobile, à suivre chacune de leurs positions » (Guibert, 1982, p. 11). Par la suite, il relate sa propre relation sexuelle avec un homme qui fait de lui sa victime. Relation sadomasochiste où le sexe de l'autre doit être désiré, imploré, adoré avant qu'il ne puisse avoir accès à lui : «tu aimerais voir mon sexe, mais il va falloir que tu le mérites, et je voudrais que tu le voies comme si tu le voyais pour la première fois, et qu'il t'éblouisse [...], que tu 
l'adores, mais pour l'instant il va falloir que tu le gagnes » (ibid, p.16). Plus tard, la position que chacun occupe s'inverse : le narrateur devient à son tour le bourreau et il lui revient de traiter son partenaire en chien. Il occupe ainsi plusieurs positions, plusieurs postures, multiplie les rôles ou les attitudes, passant des uns à leur contraire, ainsi que le remarque Heathcote, qui relève dans ce récit un «alliage de passivité et d'activité » (1995, p. 64), note que le narrateur possède «le calme, la sérénité [...] la rage et la fureur» (ibid) et souligne à juste titre que

les paires et les binarismes se multiplient au cours du roman, depuis le moment où le narrateur fait le double de son propre corps en dessinant "une sorte de cartographie" de ses membres dans le drap qu'il découpera "comme un modèle, un patron de [s] es plaisirs à venir". De même, les rites sexuels miment ses désirs d'homme célibataire, et les rites sexuels réunissent deux hommes, dont un est le double, le reflet de l'autre. Il n'y a donc rien d'étonnant à ce que le seul meuble mentionné par le narrateur soit un miroir [...] et que le seul accessoire qu'il propose à son amant soit "un miroir carré, sans cadre, sans ornement" dont ils se serviraient pour refléter, pour redoubler leurs plaisirs. (ibid)

On le comprend, ici comme dans La Mort propagande, le narrateur n'a de cesse de représenter le corps jouissant dans ce qui relève d'un déploiement de situations qui refusent et empêchent toute fixation. Bien sûr, le corps présenté, la jouissance recherchée, exprimée, textualisée, sont ceux d'un homosexuel... Mais d'un homosexuel pluriel, qui cherche à « élaborer un "je" pluriel» (Pratt, 1995, p. 74) par le pouvoir libérateur de la pornographie, cette dernière permettant, selon Leo Bersani, de briser «le moi fixe et son corps idéologique en des morceaux indistincts et communs » (ibid, p. 78). L'écriture de Guibert - la formule de Sylvain Desmille est pertinente — 
consiste bien en «une mise en vertige du je initial» transformant le narcissisme «traditionnel » en un « narcissisme pervers » qui vise non pas à chercher son reflet, à le contempler mais «qui détourne son reflet de soi pour l'afficher aux yeux des autres» (Desmille, 1989). Le corps à jouir guibertien, monstrueux en ce sens qu'il (se) donne à jouir et à voir, est aussi, par-là même, pourrait-on dire, un corps spéculaire, un corps en fuite, insaisissable. Sa construction dans l'espace littéraire est une manière d'échapper aux représentations caricaturales ou schématiques de l'homosexuel ou de l'hétérosexuel, de l'actif et du passif. Une autre illustration, pour terminer cette évocation du corps jouissant, se situe dans « $\mathrm{Au}$ musée Grévin » (Guibert, 1991c, p. 255-256), texte datant de 1978. Le narrateur se met en scène dans une série de postures où il prend la place de statues de cire :

J'ai décidé de me photographier dans certaines scènes. Donc je prends la place de Marat dans sa baignoire [...]. Je prends la place de Marie-Antoinette se pâmant en voyant à sa fenêtre la tête de la princesse de Lamballe au bout d'une pique. [...] Enfin, comme Louis XVIII dans la prison du Temple, je me cabre sur son lit de camp, le corps dressé par un cauchemar [...]. (ibid)

Le corps n'est pas présenté ici en état de jouissance, mais le narrateur semble jouir des scènes qu'il lui donne à jouer. « Hervé nous a quittés, il est passé du côté des mannequins de cire » (ibid), déclare la directrice du musée. Ces jeux sont bien source de jouissance en ce qu'ils permettent au corps de mimer ce qu'il n'est pas, en ce qu'il se donne à voir, à photographier en un autre: ils permettent au corps de s'échapper et à la photographie de saisir ce dessaisissement de soi. Offert et inaccessible : voilà ce qu'est le corps guibertien. Déjà souffrant et jouissant de cette souffrance commandée, recherchée, de 
cette perte programmée afin d'explorer les frontières poreuses qui séparent ou réunissent jouissance et douleur. Un corps fantôme avant la lettre, spectral, c'est-à-dire n'occupant jamais une posture déterminée et arrêtée... Et cette attitude se prolonge lorsque la douleur prend le pas sur la jouissance, lorsque le corps ne jouit plus de souffrir mais souffre de ne plus pouvoir jouir, comme je vais maintenant tenter de le montrer.

\section{Vers un corps fantôme : le corps malade}

Si l'on fait abstraction du roman sadien de 1987 intitulé Vous m'avez fait former des fantômes, que l'on peut lire comme une parenthèse dans l'économie générale de l'œuvre de Guibert et qui relate le dressage d'enfants par une bande mafieuse afin qu'ils soient torréés par des «infanteros » (avec tout ce que cela suppose de scènes centrées sur le corps comme objet de jouissances, de tortures, de fantasmes...), l'écriture du corps, depuis 1982, avait tendance à s'assagir dans les romans de l'écrivain. Certes, il était toujours présent, dans Des aveugles, où l'absence de la vue décuple les autres sens des personnages et notamment leur toucher, ou encore dans Mes parents, où le narrateur tente de libérer son corps de l'emprise de son père et de sa mère et s'initie aux premières amours masculines. On retrouve dans Les Gangsters (1988), à l'évocation du zona qui touche le narrateur, une fascination pour le corps, pour la peau, qui nous rappelle certains passages de La Mort propagande. Guibert s'acharne toujours à noter les moindres détails, les plus petites évolutions qui viennent stigmatiser la chair :

J'ai un zona. Piqûres d'antibiotiques, calmants à base d'opium et de belladone, rien n'apaise mes douleurs. Elles se relacent et 
s'entrecroisent, comme un feuilleté d'un raffinement diabolique. La crampe la plus sourde menace le tréfonds d'implosion imminente. La plus franche est une sensation d'éventration tantôt verticale, tantôt horizontale, une seule plaie énorme qui traverse le bassin, si fraîche qu'elle n'a laissé aux chairs le temps de se ressouder, chaque mouvement nargue les sutures. (1988b, p. 8)

Mais le corps subit désormais plus qu'il ne provoque les situations dans lesquelles il se trouve: " on aurait pu me trépaner, me planter des seringues dans le ventre et dans les yeux, j'aurais juste serré les dents, j'avais lancé mon corps dans quelque chose qui le dépossédait apparemment d'une volonté autonome » (1990a, p. 198), déclare Guibert dans À l'ami qui ne m'a pas sauvé la vie. Dans cette même optique, Gérard Danou définit quant à lui le corps souffrant comme « un corps éprouvé [...] [qui] est généralement subi passivement plutôt que mis en relief comme manifestation éclatante de la vie dans le bruissement des organes » (1994, p.11). L'antonyme, donc, d'un corps jouissant qui ne bruisse, lui, que par ses organes.

Si la maladie qu'est le sida amène Guibert à poursuivre l'histoire de son corps, si elle l'amène même à radicaliser et à intensifier cette exposition de soi, les modalités explorées restent identiques à celles déjà évoquées dans l'étude de $L a$ Mort propagande. Le corps souffrant qui se montre, qui s'écrit, va jusqu'à livrer ce qui se passe en son for intérieur. Les analyses et les rapports médicaux sont fondus dans la trame narrative d'À l'ami, ils sont livrés au lecteur, qui prend ainsi connaissance du mal qui ronge le narrateur, qui mesure, avec lui, la distance ténue le séparant de la mort. «Le processus de détérioration amorcé dans mon sang se poursuit de jour en jour, assimilant mon cas pour le moment à une leucopénie. Les dernières analyses, datées du 18 novembre, me donnent 
368 T4, un homme en bonne santé en possède entre 500 et $2000 »$ (Guibert, 1990a, p. 13). À ce propos, Guibert confie : « Raconter le processus de détérioration de son sang va au-delà de parler de son corps intime, c'est parler de l'intérieur de son corps. C'est un livre beaucoup plus impudique que tous mes livres dits érotiques»(1990b). Ce que Guibert semble avoir oublié, c'est que l'intérieur de son corps a déjà été donné en pâture aux lecteurs dans ses premiers écrits. Mais là où il $\mathrm{a}$ raison, c'est que les révélations faites ici n'engagent pas seulement le corps et sa puissance de représentation sexuelle mais bien plutôt la fragilité de ce qui le retient à la vie. Et c'est dans cette exposition-là que réside toute l'impudeur «apparente» d'un tel livre. Le corps souffrant est un corps médicalisé, offert aux regards de tous : médecins, radiologues, infirmiers, autres patients dans les salles d'attentes. C'est un corps qui, «lancé dans les circuits médicaux, perd toute identité, ne reste plus qu'un paquet de chair involontaire, brinquebalé par-ci par-là, à peine un matricule, un nom passé dans la moulinette administrative, exsangue de son histoire et de sa dignité » (Guibert, 1990a, p. 32). Le corps semble alors pris dans un double engrenage qu'il ne maîtrise plus. Celui tout d'abord de la maladie, qui dépossède le narrateur de son corps, qui fait de lui un vieillard et l'amène à s'identifier à son père ou à sa grand-tante :

Un corps de vieillard avait pris possession de mon corps d'homme de trente-cinq ans, il était probable que dans la déperdition de mes forces j'avais largement dépassé mon père qui vient d'avoir soixante-dix ans, j'ai quatre-vingt-quinze ans, comme ma grand-tante Suzanne qui est impotente [...]. (Guibert, 1991d, p. 10) 
L'autre engrenage - que suggérait une citation précédente, selon laquelle Guibert avait lancé son corps dans quelque chose qui le dépossédait d'une volonté autonome -, est celui du corps médical, qui amène le corps du narrateur, jusque-là rebelle, transgressif, à devenir, selon l'expression de Foucault, un « corps docile »: « Est docile un corps qui peut être soumis, qui peut être utilisé, qui peut être transformé et perfectionné » (1975, p. 137).

C'est à ce moment-là que Guibert décide d'emprunter les détours d'une écriture autofictionnelle pour se dire ou, plutôt, pour se dire différemment: puisque son corps lui échappe, puisque son corps lui est volé, puisque « [l]a maladie mortelle introduit une distance qui n'est guère éloignée d'une certaine schizophrénie » (Bordas, 1999), Guibert doit en passer par une mise en fiction de soi pour tenter de saisir ce qui lui échappe désormais: "La mort ne peut se contempler ou s'écrire que grâce à des détours qui font que tout de suite vous retombez dans le fictif, le faux ou le délirant» (Hill, 1995, p. 93). La fiction, comme un voile pudique sur l'impudeur de cette mort s'écrivant et se livrant en direct... Le corps de Guibert devient le lieu d'une fracture intérieure, d'une fracture autobiographique, source d'une effroyable douleur. Cette distanciation introduite par la mise en fiction de soi et le recours au genre du «roman» (cette étiquette générique, associée à l'utilisation du nom de l'auteur, est utilisée pour la première fois chez Guibert avec À l'ami qui ne m'a pas sauvé la vie) se comprend comme la manifestation des difficultés du narrateur à se reconnaître dans son enveloppe corporelle. Pour Derek Duncan, «le sida a ruiné non seulement le corps de Guibert, mais aussi le désir et la capacité de se percevoir en transformant le corps en lieu d'altérité absolue » (1995, p. 107). 
Le corps jouissant était déjà, nous l'avons dit, un corps en fuite. Il en est de même pour le corps souffrant : il est par définition fuyant, ce qui m'échappe, que je ne comprends pas et ne saisis plus. C'est la raison pour laquelle le corps souffrant se trouve être à l'image du corps jouissant, lui aussi toujours médiatisé. Guibert sera, dès 1990, constamment « animé du désir de filmer ses examens médicaux, ses séances de massage et même ses opérations. [...] Guibert vit entouré de miroirs, des miroirs qui lui renvoient l'image toujours passionnante de lui-même, des miroirs qui le préviennent et l'avertissent» (Bordas, 1999) de l'arrivée de la mort: "J'ai senti venir la mort dans le miroir, dans mon regard dans le miroir » (Guibert, 1990a, p. 15).

La plus grande manifestation de cette médiatisation du corps s'accomplira dans le projet cinématographique de l'auteur, La Pudeur ou l'Impudeur, qu'annonçait déjà, de manière prémonitoire, La Mort propagande: «Me donner la mort sur scène, devant les caméras » (Guibert, 2009a, p. 10). Le titre pose la question: ce film qui donne à voir le corps souffrant dans des scènes parfois très difficilement supportables, dans lequel l'auteur va jusqu'à filmer une tentative de suicide par empoisonnement, est-il le comble de l'impudeur du corps ? Ou alors la mise en fiction de ce corps mourant par le truchement de la caméra, qui impose par essence une mise en scène de ce qui est saisi (cadrage, répétition de la scène filmée, etc.), ne doit-il pas se concevoir comme une représentation pudique de ce qui arrive à Guibert ? Il n'y a bien évidemment pas de réponse, la question reste en suspens et laissera le spectateur libre de son interprétation.

Une des scènes emblématiques est, selon moi, celle de la tentative de suicide. Il s'agit en fait d'une fausse tentative de 
suicide, comme nous l'apprendrons en 2001, à la sortie du journal de l'auteur, Le Mausolée des amants :

Avant-hier (le temps de s'en remettre), j'ai mimé mon suicide devant la caméra. Voilà une prise que je ne saurais pas refaire, sauf pour de vrai [...]. Je filmais le simulacre de mon suicide, inventant sur-le-champ, dans le champ, le coup de la roulette russe truquée avec les verres [...]. J'ai inventé la suite, par mon jeu. (Guibert, 2001, p. 414)

Cette scène participe à la fois du projet de tout montrer, mais aussi d'instaurer une dialectique entre le fictif et le factuel et d'ériger ce jeu en parangon créatif. La dernière phrase de l'extrait cité fait intervenir le personnage / acteur Guibert. Contrairement aux autres événements filmés, cette tentative de suicide est une "première ", ce n'est pas une expérience qui a été préalablement vécue. Cependant, plus qu'une "fiction», nous pouvons envisager cette scène comme une prémonition ou, plus précisément, une répétition (consciente ou non) du véritable suicide de Guibert. Dans Le Protocole compassionnel, il écrivait : «c'est quand ce que j'écris prend la forme d'un journal que j'ai la plus grande impression de fiction» (1991d, p. 87). L'effet est ici inverse : cette «fiction» lui donne une grande impression de vérité. D'ailleurs, l'auteur dit être sorti de cette expérience "épuisé » et "modifié », c'est dire que le leurre a pris une telle ampleur qu'il a fini par devenir le double de la réalité. Le film implique le sujet à un tel point que le trucage trompe Guibert lui-même, pris au piège de sa propre machination. Il tente ici de parachever son œuvre. Pour se saisir entièrement, il faut aussi se saisir mort, se faire autothanatographe, c'est-à-dire évoquer l'intimité de la mort elle-même, sans pudeur, dire l'ultime cri du corps. 
Alors, le corps souffrant est toujours le même et déjà autre. Il est toujours un corps offert, un corps ouvert, exposé, s'exposant sans complaisance. Il est toujours monstrueux, objet du regard. Mais son exposition s'effectue dans une mise en fiction progressive, fiction qui constitue l'écran, la distance nécessaire à l'auteur pour essayer de se saisir. Cependant, le corps souffrant est différent. Il impose un saut dans le temps, il fait du jeune homme un vieillard et Guibert se projettera, se transposera d'ailleurs sous les traits d'un octogénaire dans un de ses derniers textes, Mon Valet et moi (Guibert, 1991e). C'est, semble-t-il, la raison pour laquelle il filme ses grand-tantes dans La Pudeur ou l'Impudeur. Il s'y reconnaît, elles sont un miroir de sa sénescence anticipée. Il est déjà autre, parce que désérotisé — du moins partiellement —, se retrouvant frustré ou jouissant dans des situations dans lesquelles Thanatos prend le dessus sur Éros :

Cette ébauche de baise me semblait sur l'heure d'une tristesse intolérable, j'avais l'impression que Jules et moi nous étions égarés entre nos vies et notre mort, et que le point qui nous situait ensemble dans cet intervalle, d'ordinaire et par nécessité assez flou, était devenu atrocement net, que nous faisions le point, par cet enchaînement physique, sur le tableau macabre de deux squelettes sodomites. Planté au fond de mon cul dans la chair qui enrobait l'os du bassin, Jules me fit jouir en me regardant dans les yeux. C'était un regard insoutenable, trop sublime, trop déchirant, à la fois éternel et menacé par l'éternité. (Guibert, 1990a, p. 156)

Il est déjà autre parce qu'ailleurs, comme le suggèrent le passage qui vient d'être cité mais aussi la dernière image de son film testament. Guibert, d'abord à sa table de travail, prend congé de la caméra, du spectateur, s'efface, fantomatique, et laisse entendre le cliquetis d'une machine à écrire inscrivant sur du papier les derniers mots d'une histoire qu'il sait terminée. 


\section{Le corps guibertien, un corps politique?}

J'ai tenté de montrer jusqu'ici que le corps guibertien, qu'il soit jouissant ou souffrant, est un corps appelé à s'écrire et à se montrer. L'œuvre de Guibert, le rapide parcours entre les deux extrêmes de son travail que je viens d'effectuer en témoigne, est l'histoire d'un corps, incontestablement. D'un corps fortement sexué, s'exhibant dans ses pratiques homosexuelles. C'est ce qui fait dire à l'écrivain, dans un livre qu'il consacre à la photographie en 1981, L'Image fantôme :

la plupart de mes récits suintent l'homosexualité. [...] Si je masquais mon désir, si je lui ôtais son genre, si je le laissais dans le vague, comme d'autres l'ont fait plus ou moins habilement, j'aurais l'impression d'affaiblir mes récits, de les rendre lâches. Ce n'est même pas une affaire de courage (je ne milite pas), il en va juste de la vérité de l'écriture. (1981, p. 89)

Notons rapidement que ce propos ne peut que partiellement être attesté par une lecture attentive de L'Image fantôme et du désir tel qu'il s'exprime dans cet ouvrage. Alex Hugues a montré, dans une analyse pertinente (1999, p. 53), que le désir exprimé ici est souvent moins un désir homosexuel qu'un désir indéterminé ou un désir homosexuel indéchiffrable. Elle se base pour cela sur l'étude d'un des récits, intitulé «L'image cancéreuse », qui compose le recueil. Guibert, ne pouvant se résoudre à se séparer d'une photographie ayant entrepris son processus de dégradation et représentant un jeune homme blond, " androgyne », dont on ne montre ni le corps, ni le sexe, rapporte comment il en vient à tenter son incorporation :

Puis, quelque temps, je me décidai à le porter directement sur moi, à même ma peau, à même mon torse, en l'y attachant avec des bandes et des élastiques. Il me raidissait, il me faisait 
comme un corset sous ma chemise. Je ne pouvais plus le voir, il m'embrassait le ventre, j'imaginais qu'il s'agrippait à moi comme un enfant endormi, et le contact du papier n'était plus froid contre ma peau, il s'amollissait, je le baignais de ma sueur et de ma crasse. Il était comme un second frère mort attaché à moi, il était mon hétéradelphe. (ibid. p. 168-169)

Cette image devient alors illisible et son caractère (homo)érotique initial vient à disparaître. Guibert n'incorpore plus un jeune homme blond androgyne, mais vient à le transformer en un autre lui-même, métamorphosant l'amour du jeune homme en amour de son double et donc de soi. Guibert instaure là un jeu autour de l'indétermination et de l'illisible, envisagés comme objets de ses désirs, contrecarrant l'idée que seules les images explicitement homosexuelles l'attireraient.

D'ailleurs, dix ans plus tard, dans une des dernières interviews qu'il donne, Guibert a des propos tout à fait différents quant au caractère homosexuel de son travail :

C'est comme le mot homosexualité, pour moi c'est un mot qui n'a jamais eu vraiment un rapport avec moi, bizarrement, alors qu'il en a évidemment un, mais je ne vois pas les choses comme ça, ce n'est pas la façon dont je vis, c'est pas la façon dont je me sens, j'ai l'impression que je suis ailleurs que dans ces... (1991a)

Guibert ne conclut pas son propos et laisse en suspension la définition de sa sexualité telle qu'elle se donne à lire à travers son corps textualisé. Entre la revendication - dans un sens apolitique du terme - du caractère homosexuel de son œuvre et l'évitement de cet « enfermement » ou du caractère restrictif que pourrait connoter l'adjectif, où situer le corps de Guibert?

Les critiques qui se sont penchés sur cet aspect précis de l'œuvre de Guibert sont d'accord. Je le disais dès mon introduction, l'impression qui domine est celle des derniers 
propos de l'écrivain que je viens de rapporter : c'est un corps " toujours ailleurs, il est lié à tous les ailleurs du monde et à vrai dire il est ailleurs que dans le monde» (Foucault, 1966). Guibert, en fait, ne refuse pas le qualificatif d'homosexuel. Ce que cherche à souligner l'auteur est précisément «le peu de sens qu'une définition sexuelle retient face à la multiplicité des relations, d'expériences et d'identifications qu'elle est censée désigner » (Pratt, 1995, p. 70). Accepter ce qualificatif serait se conformer et conformer son corps à ce que le système idéologique détermine par là. Or Guibert se situe davantage dans une volonté de pervertir, de transgresser cette idéologie et de proposer une identité toujours plurielle, donc insaisissable et en ce sens subversive. Écrire l'histoire de son corps, la fiction de son corps jouissant et souffrant, aurait été pour lui une manière de lutter contre les fictions idéologiques, sociales, culturelles que l'on assigne généralement à tel ou tel corps sexuellement déterminé et qu'il considère comme autant d'aliénations. Toujours selon Murray Pratt, «[1]es commentateurs de Guibert conviennent qu'il est important de rejeter l'image du Narcisse silencieux, tout comme celui du pervers duplice. Plutôt que de reproduire de tels stéréotypes homophobes, qui servent d'un côté à réduire l'attrait de l'auteur et de l'autre à en construire un épouvantail marginalisé » (Pratt, 1997, p. 134), il s'agit de dire en quoi l'écriture du corps de Guibert se situe ailleurs.

Guibert, dans ses représentations du corps, cherche selon moi à échapper à la Loi. Il n'impose pas une nouvelle image mais plutôt se soustrait à celles qui existent. L'image de son corps est une image qui résiste. Tout d'abord, les représentations qu'il propose sont, selon Michael Worton, « une interrogation de la masculinité telle qu'elle a été socialisée. [Ses 
textes] contestent la prétendue cohérence du corps masculin, substituant au corps phallique (et centré sur le pénis) un corps éclaté, qui est aussi un corps écrit » (1997, p. 72). Le corps de Guibert est en effet un corps métonymique où chaque centimètre carré doit exulter. Il est un corps tendu, raide mais aussi malléable, offert. Il est ce qui donne et qui reçoit. Il est toujours l'autre, l'irréductible, celui auquel je ne peux pas m'identifier. Les excès écrits de son corps, les scènes vécues ou fantasmées dans lesquelles il l'engage, le redoublement médiatisé de ses jouissances ou de ses souffrances, la proximité avec la mort qu'il nous amène à partager, le morcellement métonymique auquel il le soumet désorganisent, voire interdisent toute identification. L'écriture du corps guibertien est une tentative de déjouer toute convocation identitaire. Le désir de transparence qui est le sien, et ce, dès le début de son œuvre, le dévoilement impudique auquel il se livre, peuvent se concevoir comme la volonté de faire du corps un corps fantôme, c'est-à-dire non pas seulement celui qui est appelé à disparaître à cause de la maladie qui l'anéantit, le plonge dans le néant, mais celui qu'on ne peut saisir du fait justement de cette transparence recherchée et atteinte. Le monstrueux, c'est toujours l'autre. On le refuse, on le réfute. On se désidentifie à lui. Rien ne prend sur lui. Il n'appartient pas à un système, quel qu'il soit; il s'en exclut de lui-même comme pour s'offrir sa propre liberté d'être au monde.

Dans Le Protocole compassionnel, le narrateur retrouve dans ses relations avec son médecin, Claudette Demouchel, un érotisme perdu par ailleurs. Les longs examens qu'elle lui fait subir réveillent une certaine sensualité, une certaine suavité. Dans un entretien, Guibert déclare qu'à travers elle, «qui était très brutale au début, un peu masculine même, et qui se révélait 
de plus en plus douce et féminine, [il a] découvert une espèce d'attirance pour les femmes, qu'[il] n'avai[t] jamais eue. [...] C'est problématique pour un pédé de ne plus pouvoir supporter les hommes » (Guibert, 1991f). Dans son tout dernier texte, Le Paradis, Guibert va jusqu'à "s'offrir» une aventure hétérosexuelle. Le narrateur, Hervé Guibert, affaibli par une maladie qui n'est pas le sida, relate ses ébats sexuels avec Jayne, une ex-championne de natation :

[...] il nous fallait ça pour faire l'amour, et le pistolet lui entrait de plus en plus facilement dans le con, je l'y enfonçais de plus en plus profondément, jusqu'à la détente, Jayne haletait de plus en plus fort, j'avais de plus en plus hâte de la farcir avec mon foutre, et nous jouissions ensemble de plus en plus vite, de plus en plus souvent, comme deux fous. (1992, p. 19)

Dans ce dernier roman, le corps du narrateur se déprend de tout ce qui le détermine socialement: il n'a plus le sida, il n'est plus homosexuel. Il ne s'agit que de s'échapper, le temps d'un livre, de la maladie qui le condamne et de l'identité à laquelle on l'a réduit. Toujours ailleurs, en défaut de lui-même comme pour brouiller les paradigmes actif / passif, viril / non viril, hétérosexuel / homosexuel. « Le texte guibertien est toujours au moins double ; c'est ainsi que Guibert rend au corps sa matérialité complexe », nous dit encore Worton. Et de poursuivre : «Ses textes sont en mouvement perpétuel, passant sans cesse et sans prévenir d'un registre à un autre, d'une syntaxe à une autre, refusant ainsi la fixité de l'organisation génitale prônée par la société moderne qui a tendance à entraver toute tentative d'écrire le corps » (Worton, 1997, p. 73).

Il y a, chez Guibert, ce qui est de l'ordre d'une dialectique du corps: jouissant / souffrant, pudique / impudique, monstrueux / fantomatique. S'il est un motif qui hante 
l'œuvre de l'écrivain, c'est pour mieux affirmer son insaisissabilité. Pas de quête, mais une fuite. Une volonté de déconstruire tous les repères, d'annihiler les frontières. Guibert se sera acharné à n'être jamais où il devait être, à ébranler les codes, à échapper aux définitions, aux repères homos ou hétéronormés. Plus qu'à une simple thématique, Guibert, à travers la représentation du corps, se sera livré à une véritable expérience sur la mise en scène du «moi» en tentant de repousser toujours plus loin les limites du dicible et du montrable. En paraphrasant le peintre Réquichot, nous pouvons dire qu'il n'écrivait pas pour faire une œuvre, mais pour savoir jusqu'où son œuvre pouvait aller, jusqu'où le corps / sujet pouvait s'investir dans l'espace littéraire. Aller au bout d'un dévoilement de soi, c'était pour l'écrivain faire en sorte qu'il n'y ait plus de secrets, ou alors les faire circuler, comme il aimait à le faire. Et «son secret, le grand secret que toute son œuvre répète sans cesse, est que tous les genres sont suspects et que ce n'est qu'en gommant toute distinction générique que l'on construira un érotisme satisfaisant et une ontologie de l'être authentique » (Worton, p. 75).

\section{Bibliographie}

BARthes, Roland. (1973), Le Plaisir du texte, Paris, Seuil, coll. « Points littérature ».

BORDAS, Éric. (1999), « Du corps souffrant à l'âme condamnée. Le Protocole compassionnel d'Hervé Guibert», Dalhousie French Studies, ${ }^{\circ} 46$. 
Butler, Judith. (2012), Défaire le genre, Paris, Editions Amsterdam.

Danou, Gérard. (1994), Le Corps souffrant, Seyssel, Champ Vallon.

DESMILLE, Sylvain. (1989), « Le double visage d'Hervé Guibert», Gai pied hebdo, ${ }^{\circ} 364,6$ avril.

DunCAN, Derek. (1995), «Gestes autobiographiques : le sida et les formes d'expression artistiques du moi », dans JeanPierre Boulé, Nottingham French Studies, vol. 34, n 1 , printemps.

FouCAUlT, Michel. (1975), Surveiller et punir, Paris, Gallimard.

—. (2009 [1966]), Le Corps utopique. Les Hétérotopies, Fécamp, Lignes.

GUIBERT, Hervé. (2009a), La Mort propagande, Paris, Gallimard, coll. « L'arbalète ».

-. (2009b), La Pudeur ou l'Impudeur, BQHL.

-. (2001), Le Mausolée des amants, Paris, Gallimard.

—. (1992), Le Paradis, Paris, Gallimard.

- (1991a), "Pour répondre à quelques questions qui se posent... », entretien avec Christophe Donner, La Règle du jeu, vol. $3, \mathrm{n}^{\circ} 7$, mai.

—. (1991b), "Hervé Guibert et son double», propos recueillis par Didier Eribon, Le Nouvel Observateur, 18-24 juillet.

-. (1991c), La Mort propagande et autres textes de jeunesse, Régine Deforges, coll. « Le livre de poche».

—. (1991d), Le Protocole compassionnel, Paris, Gallimard. 
—. (1991e), Mon Valet et moi, Paris, Seuil.

—. (1991f), «Je n'ai jamais autant aimé la vie», entretien avec Françoise Tournier, Elle, 11 mars.

—. (1990a), À l'ami qui ne m'a pas sauvé la vie, Paris, Gallimard.

-. (1990b), «La vie sida», entretien avec Antoine de Gaudemar, Libération, $1^{\mathrm{er}}$ mars.

-. (1988a), "Les aveux permanents d'Hervé Guibert », propos recueillis par Antoine de Gaudemar, Libération, 20 octobre.

—. (1988b), Les Gangsters, Paris, Minuit.

—. (1987), Vous m'avez fait former des fantômes, Paris, Gallimard.

—. (1986), Mes parents, Paris, Gallimard.

—. (1985) Des aveugles, Paris, Gallimard.

—. (1982), Les Chiens, Paris, Minuit.

—. (1981), L'Image fantôme, Paris, Minuit.

Heathcote, Owen. (1995), «Les Chiens d'Hervé Guibert: analyse d'une plaquette pornographique », dans Jean-Pierre BOULÉ, op. cit.

HILL, Leslie. (1995), « Écrire - la maladie (à propos de quelques textes d'Hervé Guibert) », dans Jean-Pierre Boulé, op. cit.

Hugues, Alex. (1999), Heterographies, Sexual Difference in French Autobiography, Oxford, Berg.

LEIRIS, Michel. (2004), «De la littérature considérée comme tauromachie », dans L'Âge d'homme, Paris, Gallimard, coll. « Folio ». 
PRATT, Murray. (1997), «L'autoreprésentation, l'écriture autre et l'ange ", dans Le Corps textuel de Hervé Guibert, ParisCaen, Minard, La Revue des Lettres Modernes.

- (1995), « De la désidentification à l'incognito : à la recherche d'une autobiographie homosexuelle», dans Jean-Pierre Boulé, op. cit.

SARKONAK, Ralph. (1997), "Une histoire de corps", dans Le Corps textuel de Hervé Guibert, Paris-Caen, Minard, La Revue des Lettres Modernes.

WiтTкоP, Gabrielle. (2001), Le Nécrophile, Paris, Verticales.

WORTON, Michael. (1997), «En (d)écrivant le corps en imaginant l'homme. Le "vrai corps" de Guibert", dans Le Corps textuel de Hervé Guibert, Paris-Caen, Minard, La Revue des Lettres Modernes.

\section{Résumé}

La pudeur et l'impudeur sont deux mouvements utilisés par Hervé Guibert pour construire une image singulière de son corps que je me propose d'examiner dans cet article. Je souhaite ainsi de mettre en évidence une politique du corps guibertien. Le corps multiple échappe à toute forme de détention, de restriction, et se construit en dehors de toute norme sexuelle. Guibert refuse les modèles hétérosexuels et homosexuels et s'ancre lui-même dans un autre espace, incertain et indéfini. 


\begin{abstract}
Modesty and Immodesty are two movements used by Hervé Guibert to build a unique picture of his body that I propose to examine in this article in order to highlight a politics of the Guibertian's body. The multiple body escapes any form of confinement, restriction and constructs itself without any sign of sexual normativity. Guibert refuses both heterosexual and homosexual models and anchors himself in another space, uncertain and undefined.
\end{abstract}

